\title{
Nasal HFOV versus nasal IPPV as a post-extubation respiratory support in preterm infants-a randomised controlled trial
}

\author{
Soutrik Seth ${ }^{1} \cdot$ Bijan Saha ${ }^{1}$ (D) Anindya Kumar Saha ${ }^{1} \cdot$ Suchandra Mukherjee ${ }^{1} \cdot$ Avijit Hazra $^{2}$ \\ Received: 25 February 2021 / Revised: 12 April 2021 / Accepted: 15 April 2021 / Published online: 23 April 2021 \\ (C) The Author(s), under exclusive licence to Springer-Verlag GmbH Germany, part of Springer Nature 2021
}

\begin{abstract}
Early and successful extubation prevents several morbidities in preterm newborns. Several secondary non-invasive respiratory modalities exist but with their merits and demerits. Given the benefits of nasal high-frequency oscillatory ventilation (nHFOV), we tried to examine whether nHFOV could reduce reintubation rates compared to nasal intermittent positive pressure ventilation (NIPPV) during the post-extubation phase in preterm infants. Stratified randomisation based on gestational age was done for 86 mechanically ventilated preterm infants between 26 and $36^{+6}$ weeks of gestation within 2 weeks of age to receive either nHFOV or NIPPV post-extubation. The main objective was to compare extubation failure within $72 \mathrm{~h}$ following extubation and secondarily feed intolerance, intraventricular haemorrhage (IVH) (> grade 3), composite bronchopulmonary dysplasia (BPD)/mortality, composite duration of oxygen supplementation/ventilation support and $\mathrm{SpO} 2 / \mathrm{FiO} 2$ ratio. No statistical difference was noted for primary outcome (RR $0.8,95 \%$ CI: 0.23 to $2.78 ; p=1.00$ ) and secondary outcomes. However, nHFOV appeared possibly better in respect to feed tolerance rates and $\mathrm{pCO} 2$ washout.

Conclusion: Extubation failure within $72 \mathrm{~h}$ in infants less than 37 weeks of gestation did not differ between the two groups. However, nHFOV seems promising in reducing enteral feeding issues and pCO2 elimination. Larger multicentre studies are required for exploring benefits of nHFOV.
\end{abstract}

Trial registration: www.ctri.nic.in id CTRI/2019/07/020055, registration date July 5, 2019

What is Known:

- NIPPV is superior to $n C P A P$ as a secondary mode of respiratory support.

- Synchronisation is preferred for optimum ventilation.

What is New:

- $n H F O V$, a novel non-invasive respiratory modality without need for synchronisation, appears promising as a secondary mode subject to further trials.

- It seems promising in reducing enteral feeding issues and pCO2 elimination.

Keywords $\mathrm{nHFOV} \cdot \mathrm{NIPPV} \cdot$ Extubation failure $\cdot$ Respiratory distress $\cdot$ Preterm $\cdot$ Neonates

Communicated by Daniele De Luca

Bijan Saha

bijansaha18@gmail.com

Soutrik Seth

soutrik.nrs@gmail.com

Anindya Kumar Saha

sahaanindya09@gmail.com

Suchandra Mukherjee

drsmukherjee70@gmail.com
Avijit Hazra

deanipgmerkolkata@gmail.com

1 Department of Neonatology, Institute of Post Graduate Medical Education \& Research and SSKM Hospital, 244, A J C Bose Road, Kolkata PIN-700020, India

2 Department of Pharmacology, Institute of Post Graduate Medical Education \& Research and SSKM Hospital, 244, A J C Bose Road, Kolkata PIN-700020, India 


$\begin{array}{ll}\text { Abbreviations } \\ \text { BPD } & \text { Bronchopulmonary dysplasia } \\ \mathrm{CI} & \text { Confidence interval } \\ \mathrm{CO}_{2} & \text { Carbon dioxide } \\ \mathrm{ELBW} & \text { Extreme low birth weight } \\ \mathrm{FiO} 2 & \text { Fraction of inspired oxygen } \\ \text { FRC } & \text { Functional residual capacity } \\ \text { GER } & \text { Gastroesophageal reflux } \\ \text { HFOV } & \text { High-frequency oscillatory ventilation } \\ \text { IQR } & \text { Interquartile range } \\ \text { IVH } & \text { Intraventricular haemorrhage } \\ \text { IVH Gr3+ } & \text { Intraventricular haemorrhage } \\ & \text { more than grade } 3 \\ \text { MV } & \text { Mechanical ventilation } \\ \text { nCPAP } & \text { Nasal continuous positive airway pressure } \\ \text { nHFOV } & \text { Nasal high-frequency oscillatory ventilation } \\ \text { NICU } & \text { Neonatal intensive care unit } \\ \text { NIPPV } & \text { Nasal intermittent positive pressure ventilation } \\ \text { pCO2 } & \text { Partial pressure of carbon dioxide } \\ \text { PIP } & \text { Peak inspiratory pressure } \\ \text { PEEP } & \text { Positive end-expiratory pressure } \\ \text { RDS } & \text { Respiratory distress syndrome } \\ \text { RR } & \text { Relative risk } \\ \text { S/F ratio } & \text { Saturation to fraction of inspired oxygen ratio } \\ \text { SNIPPV } & \text { Synchronised nasal intermittent } \\ & \text { positive pressure ventilation } \\ \text { VILI } & \text { Ventilator-induced lung injury } \\ & \end{array}$

\section{Introduction}

The transition of intrauterine to extra-uterine environment is marked by complex pulmonary and haemodynamic changes which occur smoothly and uneventfully in most infants [1]. Disturbed adaptation to extra-uterine life leading to respiratory distress occurs in about 7\% of neonates [2]. Preterm neonates are at a greater risk for developing respiratory distress due to myriad of causes. Most of these infants therefore require some form of respiratory support to aid their breathing effort. With the advancement of medical research and science, it has been proved that mechanical ventilation, though the gold standard and probably the best mode of ventilation, is crippled with long-term respiratory morbidities. Therefore, in the quest for various non-invasive methods, the present day has seen a balanced arsenal of tools starting from synchronized noninvasive positive pressure ventilation (SNIPPV) to nasal continuous positive airway pressure (nCPAP), each of which has its pros and cons. The idea of this study is to highlight another brick in the wall, the nasal high-frequency oscillatory ventilation (nHFOV).

Time and again, it has been proved that the lesser the time an infant spends on invasive ventilation, the lesser the risk of chronic lung injury. Moreover, preterm infants need to tolerate extubation successfully. To date, nasal IPPV is considered the best modality post-extubation to enable infants to have a smooth transition from invasive to non-invasive modes. However, synchronization which is difficult to achieve in most cases, may provide better results [3]. The beauty of nHFOV lies primarily in the fact that it does not need synchronization [4]. Theoretically, nasal HFOV combines the benefits of both invasive high-frequency oscillatory ventilation (HFOV) and nasal continuous positive airway pressure (nCPAP) [4]. De Luca et al. suggested working parameters for nHFOV in different clinical scenarios, which need verification in adequately powered studies [4].

Few crossover and randomized control trials using nHFOV as a primary mode in RDS have been undertaken, but largescale data is still lacking [5-9]. In most of these studies, nHFOV was compared with nCPAP. nHFOV is being presently practised in some European countries, Canada and China. However, a worldwide acclamation of this novel method is yet to happen [10]. Only two studies have evaluated the efficacy of nHFOV post-extubation [11, 12]. SNIPPV might be the most effective non-invasive respiratory support modality in the immediate post-extubation phase but is not readily available [13]. Currently, a large multicentric trial is being conducted in China which aims to select the superior secondary mode of non-invasive ventilation amongst nCPAP, NIPPV and nHFOV [14].

We hypothesized that using nHFOV as a post-extubation mode will enable easy weaning of an infant from the ventilator and reduce the need for reintubation. Comparison of nHFOV with NIPPV is still in its early stages and more studies are required to reach consensus statement. Therefore, we planned to compare nHFOV versus NIPPV as a post-extubation respiratory mode in preterm neonates between 26 and $36^{+6}$ weeks gestational age admitted in a tertiary care centre. To the best of our knowledge, such a study has not yet been carried out. Given the advantages of nHFOV over NIPPV, this modality can provide an added advantage in a future newborn respiratory care.

\section{Material and methods}

\section{Trial design, settings and participants}

This randomized control trial was conducted in level III neonatal intensive care unit (NICU) in a tertiary care hospital in Kolkata, India from July 2019 to September 2020. All preterm newborns $\left(26-36^{+6}\right.$ weeks) with respiratory distress, presenting within 15 days of life requiring invasive ventilatory support for at least $12 \mathrm{~h}$ were enrolled in the study. Small for gestational age infants were identified according to Fenton's preterm growth chart [15]. Infants with major congenital anomalies or known/suspected chromosomal anomalies, 
upper airway anomalies, severe perinatal asphyxia or born outside the institute were excluded.

\section{Intervention}

Informed consent from parents was taken. Infants were intubated and put on Drager Babylog 8000 plus ventilator (Lübeck, Germany) using synchronised intermittent positive pressure ventilation with volume guarantee mode primarily for absent, weak, or intermittent spontaneous effort, frequent ( $>6$ events/h) or severe apnoea requiring positive pressure ventilation, marked retractions, severe tachypnea $>100 / \mathrm{min}$, Silverman Anderson score $>7, \mathrm{pH}<7.2$ and not improving, $\mathrm{pCO} 2>65$ on days 0 $3,>70$ beyond day 3 , shock requiring inotropic support. Extubation was done when working Pmean $<8 \mathrm{~cm} \mathrm{H}_{2} \mathrm{O}, \mathrm{FiO} 2$ $<0.3$ and tidal volume $<4 \mathrm{ml} / \mathrm{kg}$. Post-extubation respiratory support was provided in intervention (nHFOV) and comparator (NIPPV) groups, after randomization by simple online randomization done at the time of intubation. Blinding was not possible because of the nature of the study. nHFOV was provided by SLE 6000 ventilator (Surrey, UK) via Fisher Paykel FlexiTrunk ${ }^{\text {TM }}$ interface. NIPPV was given via Dragger Babylog 8000 plus ventilator (Lübeck, Germany) via Fisher Paykel FlexiTrunk ${ }^{\mathrm{TM}}$ interface. Cycling of prongs and masks was done every $4 \mathrm{~h}$. Chin straps were not used. Prong or mask size was chosen to have a snug fitting as per recommendations of Fisher Paykel FlexiTrunk ${ }^{\mathrm{TM}}$ interface. Caffeine was given to infants with birth weight less than $1250 \mathrm{~g}$ and continued till 5 days post-weaning from respiratory support. The oxygen saturation probe placement was standardized on pre-ductal location and was monitored continuously. Arterial blood gas was obtained at $12 \mathrm{~h}$ post-intervention. Infant's respiratory condition was monitored with Silverman Anderson scoring every $2 \mathrm{~h}$. Haemodynamic condition was assessed every $2 \mathrm{~h}$ in form of saturation, perfusion, and capillary refill time. Assessment for feed tolerance was done every $3 \mathrm{~h}$. Echocardiographic assessment of haemodynamics and patent ductus arteriosus and transcranial ultrasound for intraventricular haemorrhage was done as per unit protocol. Sepsis workup was done on clinical basis and based on risk factors as and when needed. Respiratory morbidity was assessed at appropriate time frame. Infants were followed up as specified in the foregoing until discharge from unit or death. Pre-extubation mode of invasive ventilation was at the treating physician's discretion. Settings used in the two arms are depicted in Table 1.

\section{Outcomes}

Primary outcome: Extubation failure within $72 \mathrm{~h}$. Secondary outcomes:

(i) Reintubation rate

(ii) Invasive ventilator free days [16]

(iii) $\mathrm{SpO} 2 / \mathrm{FiO} 2$ ratio (iv) Composite duration of oxygen supplementation/ ventilation support

(v) $\mathrm{pCO}_{2}$ and $\mathrm{pH} 12 \mathrm{~h}$ post-intervention

(vi) IVH (above grade 3) [17]

(vii) Composite bronchopulmonary dysplasia/mortality [18]

(viii) Rate of feed intolerance [19]

(ix) Time taken to full enteral feeds

(x) Pulmonary air leaks

\section{Sample size}

NIPPV failure rate in our NICU ranges between $25 \%$ and $35 \%$. From the Cochrane review by Lemyre et al. [3], we identified 5 studies with the use of non-synchronous NIPPV which had varied heterogeneity amongst the study populations in respect to gestational age but all of them belonged uniformly to preterm gestation. Kirpalani et al. studied the maximum number of infants in this context [20]. However, in their study, NIPPV was supposed to be delivered via mixed devices and due to unavailability of any Food and Drug Administration (FDA)approved synchronised devices, as acknowledged by the author, it can be considered that non-synchronised machines were used mostly. From these studies, the failure rate of NIPPV was cumulatively around $33 \%$ which, along with our internal NICU data, prompted us to consider a $35 \%$ failure rate for secondary mode of NIPPV. We, therefore, chose to study 43 subjects in each group in order to have $80 \%$ power to reduce the extubation failure rate from this baseline rate of 35 to $10 \%$ with a significance level of $0.05 \%$ (two-tailed), using uncorrected chi-square test to evaluate the null hypothesis. Sample size was calculated using PS: Power and Sample Size Calculation (Version 3.1.6, Department of Biostatistics, Vanderbilt University School of Medicine, Nashville, USA).

\section{Randomization}

Randomization was done by computer-generated random sequence number (Research Randomizer (Version 4.0)); further stratification was done based on gestational age into two subgroups $26-31^{+6}$ weeks and $32-36^{+6}$ weeks. The allocation ratio was $1: 1$ and concealment was done by using a serially numbered opaque sealed envelope. The generation of random numbers and assignment was done by a person not involved in the study. The infants and personnel could not be blinded due to the nature of intervention; however, the outcome assessor was blinded.

\section{Statistical analysis}

Analysis was done using GraphPad Prism version 7.0.0 for Windows, (GraphPad Software, San Diego, CA, USA), MedCalc for Windows, version 19.4 (MedCalc Software, Ostend, Belgium). Data was summarized by routine descriptive 
Table 1 Parameters of nHFOV and NIPPV arm used in the study (also added in online supplement)

\begin{tabular}{|c|c|c|}
\hline Parameters & nHFOV & NIPPV \\
\hline Initial & $\begin{array}{l}\text { Frequency } 10-12 \mathrm{~Hz} \text {, I:E ratio } 1: 1 \text {, amplitude } 25-35 \mathrm{~cm} \mathrm{H}_{2} \mathrm{O} \\
\quad \text { titrated based on visible chest oscillations and } \mathrm{pCO}_{2}, \mathrm{Pmean} \\
8-10 \mathrm{~cm} \mathrm{H}_{2} \mathrm{O} \text { titrated on oxygenation, } \mathrm{FiO}_{2} \text { to maintain } \mathrm{SpO}_{2} \text { at } \\
\quad 90-95 \%\end{array}$ & $\begin{array}{l}\mathrm{PIP}=2 \mathrm{~cm}_{2} \mathrm{O} \text { above the pre-extubation set } \mathrm{PIP} \text { on mechanical } \\
\quad \text { ventilation } \\
\text { Positive end-expiratory pressure }(\mathrm{PEEP})=4-6 \mathrm{~cm} \mathrm{H}_{2} \mathrm{O} \text { or iden- } \\
\quad \text { tical to PEEP during mechanical ventilation } \\
\text { Inspiratory time }(\mathrm{Ti})=0.30-0.45 \mathrm{~s} \\
\text { Pmean } 8-10 \mathrm{~cm} \mathrm{H}_{2} \mathrm{O} \\
\text { Respiratory rate }(\mathrm{RR})=40-50 \text { breaths } / \mathrm{min} \\
\text { Flow }=8-101 / \mathrm{min} \\
\mathrm{FiO} 2=\text { adjusted to maintain } \mathrm{SpO}_{2} \text { between } 90 \% \text { and } 95 \%\end{array}$ \\
\hline Weaning & $\begin{array}{l}\text { FiO2 weaned first by } 3-5 \% \text { while maintaining target saturation } \\
\text { until it reaches } 30 \% \text {, then Pmean tapered every } 6 \text { h by } 1 \mathrm{~cm} \\
\text { until } 6 \mathrm{~cm} \mathrm{H} \mathrm{H}_{2} \mathrm{O}\end{array}$ & $\begin{array}{l}\mathrm{FiO}_{2} \text { was decreased by } 3-5 \% \text { while maintaining target } \mathrm{SpO}_{2} \text { in } \\
\text { range of } 90-95 \% \text { until it reached } 30 \% \text {; then PIP was tapered } \\
\text { every } 6 \mathrm{~h} \text { by } 1-2 \mathrm{~cm} \text { till } 12 \mathrm{~cm} \mathrm{H}_{2} \mathrm{O} \text {. Subsequently, Pmean } \\
\text { lowered to } 6 \mathrm{~cm} \mathrm{H} \mathrm{H}_{2} \mathrm{O}\end{array}$ \\
\hline Discontinuation & $\begin{array}{l}\mathrm{FiO} 2<30 \%, \text { Pmean }<6 \mathrm{~cm} \mathrm{H}_{2} \mathrm{O} \\
\text { Minimal or no signs of respiratory distress and haemodynamically } \\
\text { stable for } 24 \mathrm{~h} \\
\text { Discontinued to nCPAP or } \mathrm{O}_{2} \text { or room air }\end{array}$ & $\begin{array}{l}\text { Minimal or no signs of respiratory distress on NIV pressure }(\mathrm{PIP}< \\
\left.13, \mathrm{PEEP}<5 \mathrm{~cm} \mathrm{H}_{2} \mathrm{O}\right), \mathrm{FiO}_{2}<0.3, \text { Pmean }<6 \mathrm{~cm} \mathrm{H}_{2} \mathrm{O} \text { and } \\
\text { haemodynamic stability for } 24 \mathrm{~h} \text {. } \\
\text { Discontinued to nCPAP or } \mathrm{O}_{2} \text { or room air }\end{array}$ \\
\hline Upgradation & $\begin{array}{l}\text { Pmean was increased by } 1 \mathrm{~cm} \mathrm{H}_{2} \mathrm{O} \text { at a time up to a maximum of } \\
12 \mathrm{~cm} \text { and } \mathrm{FiO}_{2} \text { increased up to } 60 \%\end{array}$ & $\begin{array}{l}\mathrm{PIP} \text { was increased up to a maximum of } 25 \text {, with simultaneous } \\
\text { increase of PEEP to a maximum of } 6 \text { and } \mathrm{FiO}_{2} \text { to } 60 \% \text { to } \\
\text { maintain target saturation. Pmean increased to } 12 \mathrm{~cm} \mathrm{H} \mathrm{H}_{2} \mathrm{O}\end{array}$ \\
\hline Failure & $\begin{array}{l}\text { Pmean }>12 \text { and } / \text { or } \mathrm{FiO}_{2}>60 \%, \mathrm{pH}<7.20 \text { and } / \text { or } \mathrm{pCO}_{2}>60 \mathrm{~mm} \\
\mathrm{Hg} \text {, frequent bradycardia }(<100 \mathrm{bpm}) \text { and desaturation }\left(\mathrm{SpO}_{2}<\right. \\
85 \%) \text { or apnoea (defined as three or more apneic episodes of } \\
\text { any degree of severity within a period of } 1 \mathrm{~h}) \text {, shock requiring } \\
\text { inotropes and Silverman Anderson score of }>6 \text { as per unit pro- } \\
\text { tocol } \\
\text { In case of failure, infants were intubated }\end{array}$ & $\begin{array}{l}\mathrm{PIP}>25, \mathrm{PEEP}>6, \mathrm{FiO}_{2}>60 \%, \text { Pmean }>12 \mathrm{~cm} \mathrm{H}_{2} \mathrm{O}, \mathrm{pH}<7.20 \\
\text { and/or } \mathrm{pCO}_{2}>60 \mathrm{~mm} \mathrm{Hg} \text {, frequent bradycardia }(<100 \mathrm{bpm}) \\
\text { and desaturation }\left(\mathrm{SpO}_{2}<85 \%\right) \text { or apnoea (defined as three or } \\
\text { more apneic episodes of any degree of severity within a period } \\
\text { of } 1 \text { hour), shock requiring inotropes and Silverman Anderson } \\
\text { score of }>6 \text { as per unit protocol } \\
\text { In case of failure, infants were intubated }\end{array}$ \\
\hline
\end{tabular}

statistics, median and interquartile range for numerical variables, and counts and percentages for categorical variables. Numerical variables were compared between subgroups by Student's independent samples $t$ test if normally distributed, or by MannWhitney $U$ test if otherwise. Fisher's exact test was employed along with calculation of relative risk (RR) and 95\% confidence interval (CI) for inter-group comparison of categorical variables. Analyses were two-tailed and statistical significance level was set at $p<0.05$ for all comparisons.

\section{Ethics}

This study was carried out in accordance with the Declaration of Helsinki and was approved by the institutional ethics committee of the Institute of Post Graduate Medical Education and Research, Kolkata, India (IPGME\&R/IEC/2019/434). Written informed consent was obtained from all legal guardians before participation in the study. This trial was registered in Clinical trial registry of India (Registration number CTRI/2019/07/020055).

\section{Results}

Out of 650 total preterm live births during the study period, 112 infants were assessed for eligibility. After exclusion of 26, a total of 86 infants were subjected to stratified randomisation into one of the two groups of nHFOV and NIPPV, stratification done based on gestational age in two subgroups $26-31^{+}$ ${ }^{6}$ weeks and $32-36^{+6}$ weeks. The flow of participants in the study is summarised in Fig. 1. Both groups were comparable with the baseline variables (Table 2). The median gestation age of the infants was 31.5 weeks with a median birth weight of $1500 \mathrm{~g}$. The duration of invasive ventilation before being extubated to the respective intervention arms was a median of $29 \mathrm{~h}$ for $\mathrm{nHFOV}$ and $27 \mathrm{~h}$ for NIPPV. The results of the primary and secondary outcomes are depicted in Tables 3, 4 and 5.

There was no reduction in need of reintubation at $72 \mathrm{~h}$ between the nHFOV group (9.3\%) and the NIPPV group $(11.6 \%)(p=1.000)$. In a subgroup analysis also, statistical significance was not found for the primary outcome. Overall reintubation rate was reduced but not statistically significant (16.2 vs. 18.6\%). Composite duration of oxygen supplementation/ventilation support and $\mathrm{Spo} 2 / \mathrm{FiO} 2$ ratio was similar between the two groups.. There was a possible trend towards better $\mathrm{pCO} 2$ elimination $(p=0.097)$ and $\mathrm{pH}$ optimisation ( $p=0.073$ ) $12 \mathrm{~h}$ after the start of intervention. No differences were observed between the two groups as well as sub groups for IVH (> grade 3), air leaks, composite BPD/ mortality before discharge. There was a possible trend towards 
Table 2 Baseline variables of the enrolled subjects

\begin{tabular}{|c|c|c|c|}
\hline Variable & nHFOV $(n=43)$ & $\operatorname{NIPPV}(n=43)$ & Significance \\
\hline Age of mother, years, median (IQR) & $25(21$ to 31$)$ & $26(23$ to 30$)$ & $p=0.403$ \\
\hline Gestation, weeks, median (IQR) & $32(28$ to 35$)$ & $31(29$ to 35$)$ & $p=0.785$ \\
\hline Number of fetuses (single/twin), $n(\%)$ & $32(74.4) / 11(25.6)$ & $27(62.8) / 16(37.2)$ & $p=0.24$ \\
\hline Maternal pregnancy-induced hypertension, $n(\%)$ & $5(11.6)$ & $4(9.3)$ & $p=0.12$ \\
\hline Maternal gestational diabetes, $n(\%)$ & $6(13.9)$ & $4(9.3)$ & $p=0.5$ \\
\hline Maternal PROM, $n(\%)$ & $14(32.5)$ & $10(23.2)$ & $p=0.33$ \\
\hline Maternal antepartum haemorrhage, $n(\%)$ & $3(6.9)$ & $2(4.6)$ & $p=0.64$ \\
\hline Maternal hypothyroidism, $n(\%)$ & $2(4.6)$ & $2(4.6)$ & $p=1.00$ \\
\hline Maternal oligohydramnios, $n(\%)$ & $7(16.2)$ & $3(6.9)$ & $p=0.17$ \\
\hline Antenatal steroids (complete/incomplete), $n(\%)$ & $5(11.6) / 21(48)$ & $4(9.3) / 22(51.1)$ & $p=1.00$ \\
\hline Birth weight, grams, median (IQR) & $1500(1120$ to 2140$)$ & 1495 (980 to 2214$)$ & $p=0.47$ \\
\hline Small for gestational age, $n(\%)$ & $6(13.9)$ & $3(6.9)$ & $p=0.29$ \\
\hline Male, $n(\%)$ & $24(55.9)$ & $24(55.9)$ & $p=1.00$ \\
\hline Apgar 5 min, median (IQR) & $7(6$ to 8$)$ & $7(6$ to 8$)$ & $p=0.71$ \\
\hline Positive pressure ventilation, $n(\%)$ & $21(48.8)$ & $21(48.8)$ & $p=1.00$ \\
\hline Intubation, $n(\%)$ & $7(16.3)$ & $13(30.23)$ & $p=0.12$ \\
\hline Silverman Anderson score, median (IQR) & $4(4$ to 5$)$ & $4(3$ to 5$)$ & $p=0.472$ \\
\hline Respiratory distress syndrome, $n(\%)$ & $26(60.4)$ & $29(67.4)$ & $p=0.5$ \\
\hline Surfactant, $n(\%)$ & $25(58.1)$ & $28(65.1)$ & $p=0.5$ \\
\hline Duration of invasive ventilation, hours, median (IQR) & $29(22$ to 54$)$ & $27(14$ to 52$)$ & $p=0.222$ \\
\hline pCO2 before, mm Hg, median (IQR) & $41.3(32.02$ to 47.4$)$ & $38.9(34.1$ to 45$)$ & $p=0.86$ \\
\hline $\mathrm{pH}$ before intervention, median (IQR) & $7.349(7.268$ to 7.386$)$ & $7.328(7.27$ to 7.357$)$ & $p=0.43$ \\
\hline SNAPPE 2 scores, median (IQR) & $17(6.25$ to 35.75$)$ & $21(6.25$ to 35.25$)$ & $p=0.65$ \\
\hline Mean airway pressure before extubation, median (IQR) & $9.6(9$ to 10.6$)$ & $10(9.3$ to 11$)$ & $p=0.184$ \\
\hline FiO2 before extubation, median (IQR) & $21(21$ to 30$)$ & $21(21$ to 25$)$ & $p=0.568$ \\
\hline
\end{tabular}

Abbreviations: PROM, premature rupture of membranes; SNAPPE 2, score for neonatal acute physiology with perinatal extension 2; FiO2, fraction of inspired oxygen; $I Q R$, inter quartile range

lesser feed intolerance in the nHFOV group (37.2 vs. $58.13 \%$, $p=0.084)$. However, time to achieve full enteral feeds was similar amongst the groups as well as subgroups. We did not find any difference in the number of ventilator free days between the two groups.

\section{Discussion}

In our randomised controlled trial, there was no difference in reintubation rates within $72 \mathrm{~h}$ in nHFOV group, compared with NIPPV group. The consideration of higher NIPPV failure rates based on western literature and the desired reduction led to relatively smaller sample size estimation than ideally required for a statistical significant reduction of primary outcome. Need for mechanical ventilation was significantly reduced in many studies and two meta-analysis using nHFOV as a primary mode; however, all of them used nCPAP as the comparator arm [5, 6, 21]. nCPAP is still considered the standard of care post-extubation, while NIPPV now appears to be the better modality, with its own pitfalls [13]. nHFOV is adjudged in benchmark studies as a better respiratory support modality [4]. One RCT by Chen et al. with 206 infants showed lesser reintubation and $\mathrm{pCO} 2$ in $6 \mathrm{~h}$ time with nHFOV post-extubation against nCPAP [11]. Malakian et al., however, did not find any difference in rate of intubation within $72 \mathrm{~h}$ [9]. To the best of our knowledge, this is the first randomised controlled trial comparing nHFOV versus NIPPV as a post-extubation modality in preterm infants worldwide. The only trial (NCT02543125) comparing nHFOV with
Table 3 Primary outcome measures

\begin{tabular}{lllll}
\hline Variable & $\operatorname{nHFOV}(n=43)$ & $\operatorname{NIPPV}(n=43)$ & $\begin{array}{l}\text { Relative risk (95\% } \\
\text { confidence interval })\end{array}$ & Significance \\
\hline Reintubation within $72 \mathrm{~h}, n(\%)$ & $4(9.3)$ & $5(11.6)$ & $0.8(0.23$ to 2.78$)$ & $p=1.000$ \\
\hline
\end{tabular}


Table 4 Secondary outcome measures

\begin{tabular}{|c|c|c|c|c|}
\hline Variable & $\operatorname{nHFOV}(n=43)$ & $\operatorname{NIPPV}(n=43)$ & Relative risk ( $95 \%$ confidence interval) & Significance \\
\hline Reintubation rate, $n(\%)$ & $7(16.2)$ & $8(18.6)$ & $0.88(0.35$ to 2.2$)$ & $p=1.000$ \\
\hline pCO2 after intervention, $\mathrm{mm} \mathrm{Hg}$, median (IQR) & $33.8(29.125$ to 41$)$ & $37.9(32$ to 42.5$)$ & & $p=0.097$ \\
\hline $\mathrm{pH}$ post-intervention, median (IQR) & $7.39(7.348$ to 7.438$)$ & $7.35(7.313$ to 7.406$)$ & & $p=0.073$ \\
\hline IVH Gr3+, $n(\%)$ & $1(2.3)$ & $2(4.3)$ & $0.5(0.05$ to 5.31$)$ & $p=1.000$ \\
\hline Feed intolerance, $n(\%)$ & $16(37.2)$ & $25(58.13)$ & $0.64(0.40$ to 1.02$)$ & $p=0.084$ \\
\hline Full feed day of life, days, median (IQR) & $7(5$ to 10$)$ & $8(5.75$ to 10.25$)$ & & $p=0.503$ \\
\hline Air leaks, $n(\%)$ & $5(11.6)$ & $1(2.3)$ & $5(0.61$ to 41.06$)$ & $p=0.202$ \\
\hline Ventilator free days, days, median (IQR) & 26.54 (24.75 to 26.92$)$ & 26.67 (22 to 27.42 ) & & $p=0.944$ \\
\hline Composite BPD/mortality, $n(\%)$ & $12(27.9)$ & $15(34.8)$ & $0.80(0.426$ to 1.503$)$ & $p=0.485$ \\
\hline $\begin{array}{l}\text { Composite } \mathrm{O} 2 \text { supplementation/ventilatory } \\
\text { support, days, median (IQR) }\end{array}$ & $8(4$ to 21$)$ & $9(5$ to 18$)$ & & $p=0.944$ \\
\hline $\mathrm{SpO} 2 / \mathrm{FiO} 2$ ratio, median (IQR) & 310 (260 to 316.67$)$ & 306.67 (260 to 368$)$ & & $p=0.198$ \\
\hline
\end{tabular}

NIPPV as a post-extubation modality was initiated in China in the year 2016 and is yet to be completed. The composite duration of oxygen supplementation/ventilation support, $\mathrm{SpO} 2 / \mathrm{FiO} 2$ ratio during the intervention in both groups was also similar in the study. These, along with no difference in primary outcome can be explained by the fact that both the interventions were working at similar mean airway pressures which is unlikely to bring about a difference in oxygenation.

Assessment of extubation readiness should be done with lung mechanics, pressure time index, minute ventilation test apart from clinical tests and assessment of dynamics of biological signals. One of the key factors for successful extubation depends on the mode of respiratory support provided post-extubation, so as to keep the lungs open. Synchronised form of NIPPV seems to be the best choice but unavailability seems to be an issue. The pre-extubation mean airway pressure (MAP), FiO2 and tidal volume in volume ventilation play key roles in the success of extubation. Though failure rates increase with decreasing gestation and weight, secondary measures can be taken to sustain a successful extubation. Another factor which might help prevent extubation failure is efficacious clearance of pCO2. Our study showed possible trend towards better clearance of pCO2 and normalisation of blood gases but failed to achieve statistical significance. Mukherjee et al., Colaizy et al. and Czernik et al. also showed similar significant reductions in pCO2 [12, 22, 23]. There was a time-dependent variation in pCO2 levels in many studies probably because of different amplitudes used. In our study, we used a mean airway pressure of $10.9 \pm 2.06$ and a median amplitude of 15 which was similar to most studies done with nHFOV $[4,5,11,21,24]$. The MAP was similar in the two groups and this was an advantage since it allowed us to compare the effect of the pressure waveform and the active expiration as these seem to be the only real difference between groups. On the contrary, low MAPs in nHFOV probably failed to recruit the lung effectively.
Added advantages of nHFOV include reduced episodes of bradycardia and desaturation [25], enhanced alveolar ventilation due to better alveolar recruitment and improved functional residual capacity (FRC), theoretically lesser ventilatorinduced lung injury (VILI) [26], and reduced gastroesophageal reflux [27]. Various physiological and benchmark studies have demonstrated positive results in favour of nHFOV, viz. the feasibility of nHFOV in extreme low birth weight (ELBW) infants [28], effectiveness of different interfaces delivering nHFOV [24, 29, 30], the efficiency in eliminating carbon dioxide $\left(\mathrm{CO}_{2}\right)[12,21,26]$, the effect of different parameters and leak on $\mathrm{CO}_{2}$ removal [24, 29, 31, 32], the transmission of oscillation and tidal volume delivery in the airways [31].

In this study, we found that infants in the nHFOV arm seemed to have better feed tolerance rates and earlier full enteral feeds by 1 day. Given the advantage of nHFOV over NIPPV in the aspect of no need for synchronisation [4], no glottic constrictions during breaths [33], and an active expiration, it is not surprising that nHFOV seems to lower feed intolerance rates. Because of lack of synchronised machines from this part of the world, we need to strike a fine balance between non-invasive ventilation and feed intolerance issues, and in this regard nHFOV seems promising.

As for secondary outcomes like IVH, more than grade 3, air leaks, ventilator free days, composite BPD/mortality, composite oxygen supplementation/ventilation support, $\mathrm{S} / \mathrm{F}$ ratio did not differ much between the groups as well as subgroups. Interestingly, most studies done on nHFOV including the two meta-analysis failed to show any difference in BPD, air leaks, IVH and mortality [5-7, 9, 11, 21]. Though the literature suggests viscid secretions interfering with efficacy of nHFOV [10], we did not find this alarming side effect probably because of good nursing care and maintenance of oral hygiene while on ventilation. We cycled between short binasal prongs and mask every 4-hourly, both of which have been shown to be efficacious [24, 29-31]. However, none of the 


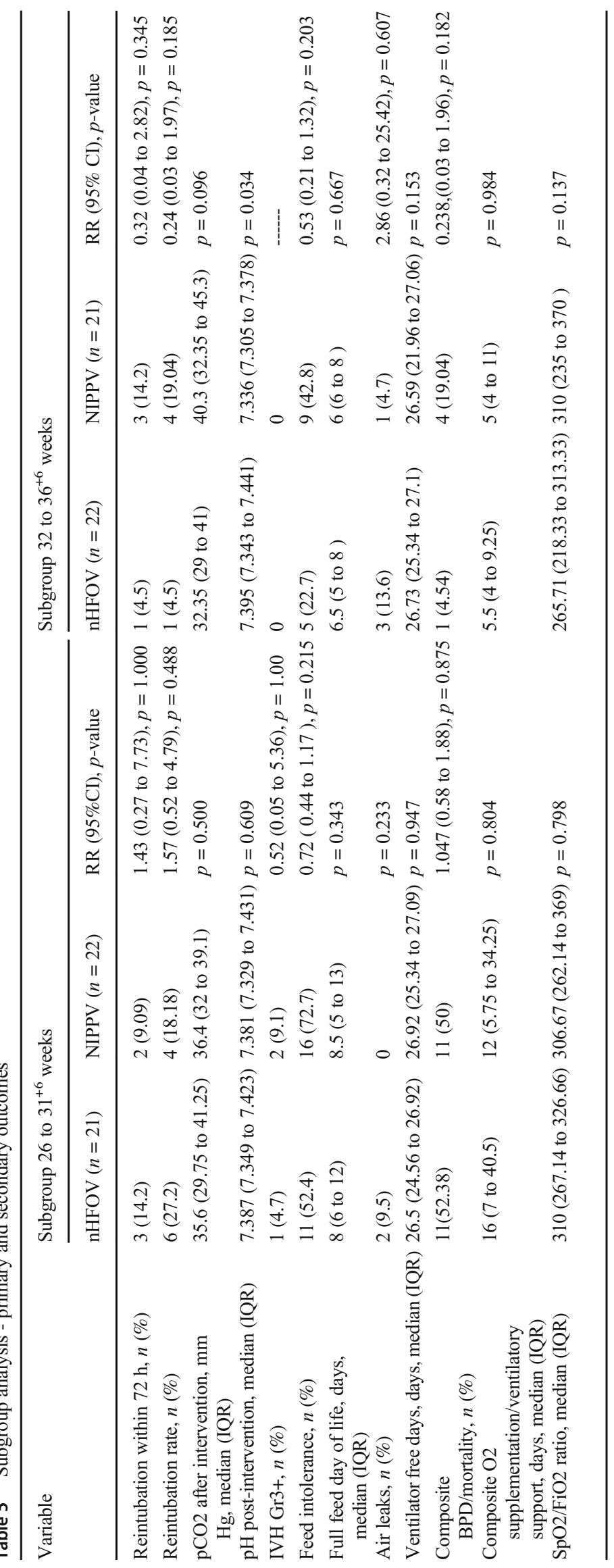




\section{CONSORT Flow Diagram}

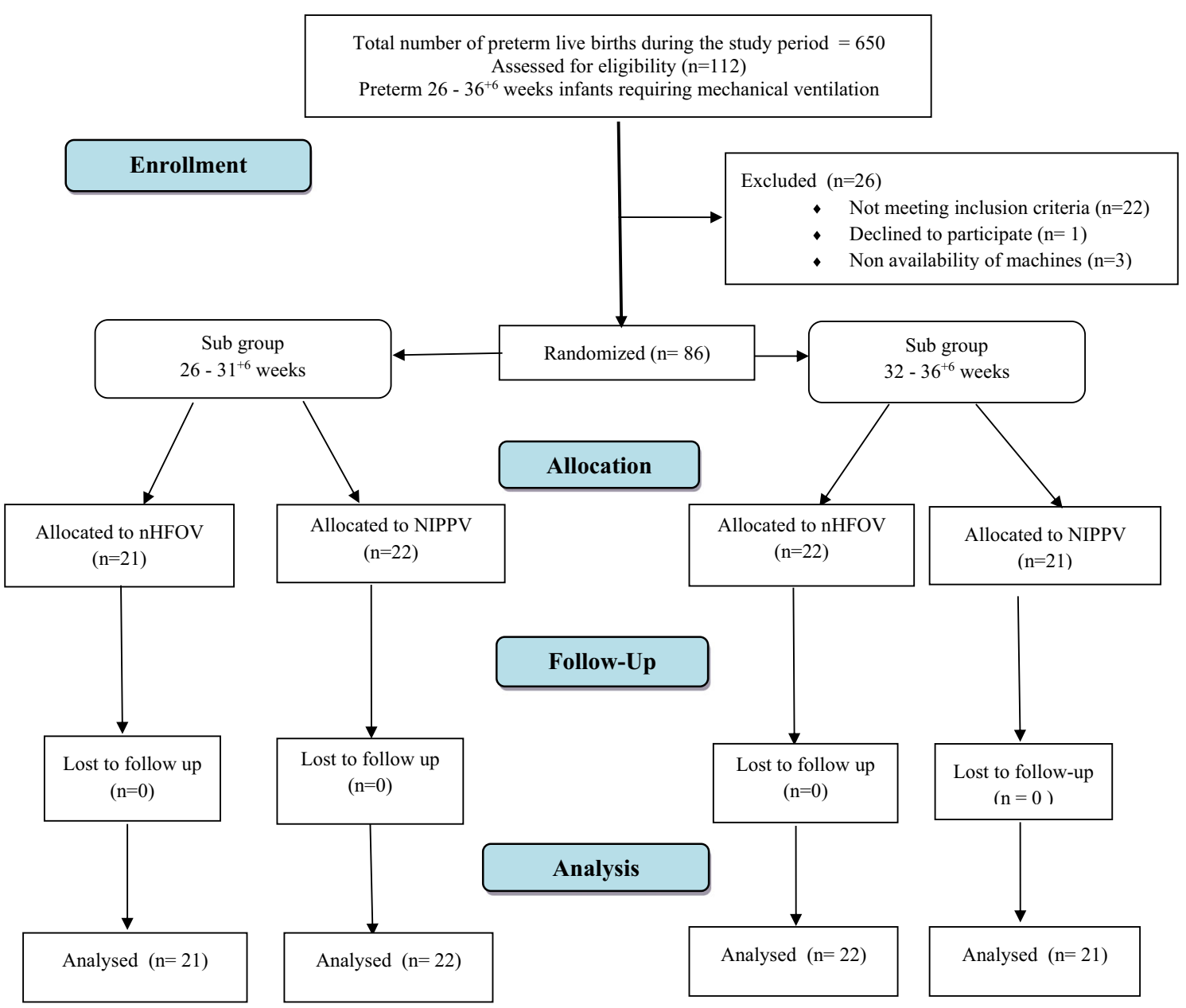

Fig. 1 Flow of participants in the study

previous studies have used both simultaneously. Maskdelivered nHFOV has more dampening effect, therefore, theoretically would require more aggressive ventilatory parameters [30]. In our case, cycling of the interfaces was done for the comfort of the infants and whether it caused a drop in efficacy of nHFOV cannot be ascertained. It will require more studies to evaluate this aspect.

The major limitations of this study are firstly, a small sample size to derive any statistical significance. The higher NIPPV failure rate consideration at the onset of the study probably led to a relatively smaller sample size estimation. Further, comfort level of infants were not quantified objectively, neither GER could be documented. Also, whether intermittent use of mask led to lesser efficacy needs to be investigated further. Moreover, long-term side effects and neurodevelopmental outcome are something to look forward to. As far as blinding was concerned, neither the infants nor the personnel were blinded. However, the person performing the final analysis was blinded to the intervention. In this regard, we acknowledge that the data compilation could have been blinded. The Pmean pressures used in nHFOV arm was possibly sub-optimal without any effective alveolar recruitment as per available evidences [4]. Unfortunately, this was done as per our pre-planned study protocol. Finally, a multicentre study is the need of the hour for formulating standard operating protocols as well as better utilisation of this novel respiratory modality.

In summary, among preterm ventilated infants, nHFOV did not bring about a significant reduction in reintubation rates within $72 \mathrm{~h}$ of post-extubation. It seemed promising in reducing feed intolerance and optimising arterial blood $\mathrm{pH}$ and $\mathrm{pCO} 2$. Most secondary outcomes, however, were similar between the two groups. Further multicentric studies need to be planned to explore further benefits of this novel respiratory support as well as formulate standard operating protocols.

Supplementary Information The online version contains supplementary material available at https://doi.org/10.1007/s00431-021-04084-1. 
Acknowledgements We would like to thank Dr. Bhupendra Kumar Gupta for generation of random sequence and assigning participants to intervention.

Authors' contributions Dr. Soutrik Seth conceptualized and designed the study, developed the protocol, patient management and prepared the first draft. Dr. Bijan Saha helped in protocol development, coordinated, supervised data collection, reviewed and revised the manuscript at all stages of its production. Dr. Anindya Kumar Saha critically reviewed and revised the manuscript. Dr. Suchandra Mukherjee helped in protocol development and critically reviewed the manuscript for improving the content. Dr. Avijit Hazra performed the statistical analysis of the data. All the authors approved the final manuscript as submitted and agree to be accountable for all aspect of the work.

Data and materials availability Available for viewing at https://drive. google.com/file/d/1ZH7jElbBkzTvwrpdQLi_AgnJ3413Aq73/view? usp=sharing

Code availability N/A.

\section{Declarations}

Ethics approval and consent to participate Informed consent was obtained from the legal guardian of all included participants.

Consent for publication Informed consent was obtained from the legal guardian of all included participants.

Conflict of interest The authors declare no competing interests.

\section{References}

1. Martin RJ, Crowley MA (2013) Respiratory problems. In: Fanaroff AA, Fanaroff JM (eds) Care of the high risk neonate, 6th edn. Elsevier Saunders, Philadelphia, p 244

2. Agarwal R, Deorari A, Paul V, Sankar MJ, Sachdeva A (2019) AIIMS Protocols in Neonatology, 2nd edn. Noble Vision, Delhi

3. Lemyre B, Davis PG, De Paoli AG, Kirpalani H (2014) Nasal intermittent positive pressure ventilation (NIPPV) versus nasal continuous positive airway pressure (NCPAP) for preterm neonates after extubation. Cochrane Database Syst Rev (9):CD003212

4. De Luca D, Dell'Orto V (2016) Non-invasive high-frequency oscillatory ventilation in neonates: review of physiology, biology and clinical data. Arch Dis Child Fetal Neonatal Ed 101:F565-F570

5. Haidar Shehadeh AM (2019) Non-invasive high flow oscillatory ventilation in comparison with nasal continuous positive pressure ventilation for respiratory distress syndrome, a literature review. J Matern Fetal Neonatal Med 7:1-10

6. Zhu XW, Zhao JN, Tang SF, Yan J, Shi Y (2017) Noninvasive high-frequency oscillatory ventilation versus nasal continuous positive airway pressure in preterm infants with moderate-severe respiratory distress syndrome: a preliminary report. PediatrPulmonol. 52(8):1038-1042

7. Iranpour R, Armanian AM, Abedi AR, Farajzadegan Z (2019) Nasal high-frequency oscillatory ventilation (nHFOV) versus nasal continuous positive airway pressure (NCPAP) as an initial therapy for respiratory distress syndrome (RDS) in preterm and near-term infants. BMJ Paediatr Open 3(1):e000443

8. Klotz D, Schneider H, Schumann S, Mayer B, Fuchs H (2018) Non-invasive high-frequency oscillatory ventilation in preterm infants: a randomised controlled cross-over trial. Arch Dis Child Fetal Neonatal Ed 103(4):F1-F5

9. Malakian A, Bashirnezhadkhabaz S, Aramesh MR, Dehdashtian M (2020) Noninvasive high-frequency oscillatory ventilation versus nasal continuous positive airway pressure in preterm infants with respiratory distress syndrome: a randomized controlled trial. J Matern Fetal Neonatal Med 33(15):2601-2607

10. Fischer HS, Bohlin K, Bührer C, Schmalisch G, Cremer M, Reiss I, Czernik C (2015) Nasal high-frequency oscillation ventilation in neonates: a survey in five European countries. Eur J Pediatr 174(4):465-471

11. Chen L, Wang L, Ma J, Feng Z, Li J, Shi Y (2019) Nasal highfrequency oscillatory ventilation in preterm infants with respiratory distress syndrome and ARDS after extubation: a randomized controlled trial. Chest. 155(4):740-748

12. Mukerji A, Singh B, Helou SE, Fusch C, Dunn M, Belik J, Shah V (2015) Use of noninvasive high-frequency ventilation in the neonatal intensive care unit: a retrospective review. Am J Perinatol 30: $171-176$

13. Ramaswamy VV, Bandyopadhyay T, Nanda D, Bandiya P, More K, Oommen VI, Gupta A (2020) Efficacy of noninvasive respiratory support modes as postextubation respiratory support in preterm neonates: a systematic review and network meta-analysis. Pediatr Pulmonol 55:2924-2939

14. Shi Y, De Luca D, (2019) NASalOscillatioN post-Extubation (NASONE) study group. Continuous positive airway pressure (CPAP) vs. noninvasive positive pressure ventilation (NIPPV) vs. noninvasive high frequency oscillation ventilation (nHFOV) as post-extubation support in preterm neonates: protocol for an assessor-blinded, multicenter, randomized controlled trial. BMC Pediatr.;19(1):256

15. Fenton TR, Nasser R, Eliasziw M, Kim JH, Bilan D, Sauve R (2013) Validating the weight gain of preterm infants between the reference growth curve of the fetus and the term infant. BMC Pediatr 13(1):92

16. Dell'Orto V, Raschetti R, Centorrino R, Montane A, Tissieres P, Yousef N, De Luca D (2019) Short- and long-term respiratory outcomes in neonates with ventilator-associated pneumonia. Pediatr Pulmonol 54:1982-1988

17. Papile LA, Burstein J, Burstein R, Koffler H (1978) Incidence and evolution of subependymal and intraventricular hemorrhage: a study of infants with birth weights less than $1,500 \mathrm{gm}$. J Pediatr 92(4):529-534

18. Jobe AH, Bancalari E (2001) Bronchopulmonary dysplasia: NICHD/NHLBI/ORD workshop summary. Am J Respir Crit Care Med 163:1723-1729

19. Moore TA, Wilson ME (2011) Feeding intolerance: a concept analysis. Adv Neonatal Care 11(3):149-154

20. Kirpalani H, Millar D, Lemyre B, Yoder BA, Chiu A, Roberts RS, NIPPV Study Group (2013) A trial comparing noninvasive ventilation strategies in preterm infants. N Engl J Med 369(7):611-620

21. Li J, Li X, Huang X, Zhang Z (2019) Noninvasive high-frequency oscillatory ventilation as respiratory support in preterm infants: a meta-analysis of randomized controlled trials. Respir Res 20:58

22. Colaizy TT, Younis UM, Bell EF, Klein JM (2008) Nasal highfrequency ventilation for premature infants. Acta Paediatr 97(11): $1518-1522$

23. Czernik C, Schmalisch G, Bührer C, Proquitté H (2012) Weaning of neonates from mechanical ventilation by use of nasopharyngeal high-frequency oscillatory ventilation: a preliminary study. J Matern Fetal Neonatal Med 25(4):374-378

24. De Luca D, Piastra M, Pietrini D, Conti G (2012) Effect of amplitude and inspiratory time in a bench model of non-invasive HFOV through nasal prongs. Pediatr Pulmonol 47:1012-1018

25. Rüegger CM, Lorenz L, Kamlin OC, Manley BJ, Owen LS, Bassler D et al (2018) The effect of noninvasive high-frequency oscillatory 
ventilation on desaturations and Bradycardia in very preterm infants: a randomized crossover trial. J Pediatr 201:269-273.e2

26. Bottino R, Pontiggia F, Ricci C, Gambacorta A, Paladini A, Chijenas V et al (2018) Nasal high-frequency oscillatory ventilation and $\mathrm{CO} 2$ removal: a randomized controlled crossover trial. Pediatr Pulmonol 53(9):1245-1251

27. Cantin D, Djeddi D, Samson N, Nadeau C, Praud JP (2018) Nasal high-frequency oscillatory ventilation inhibits gastroesophageal reflux in the neonatal period. Respir Physiol Neurobiol 251:28-33

28. Aktas S, Unal S, Aksu M, Ozcan E, Ergenekon E, Turkyilmaz C, Hirfanoglu I, Atalay Y (2016) Nasal HFOV with binasal cannula appears effective and feasible in ELBW newborns. J Trop Pediatr 62(2):165-168

29. De Luca D, Carnielli VP, Conti G, Piastra M (2010) Noninvasive high frequency oscillatory ventilation through nasal prongs: bench evaluation of efficacy and mechanics. Intensive Care Med 36(12): 2094-2100
30. Centorrino R, Dell'Orto V, Gitto E, Conti G, De Luca D (2019) Mechanics of nasal mask-delivered HFOV in neonates: a physiologic study. Pediatr Pulmonol 54(8):1304-1310

31. De Luca D, Costa R, Visconti F, Piastra M, Conti G (2016) Oscillation transmission and volume delivery during face maskdelivered HFOV in infants: bench and in vivo study. Pediatr Pulmonol 51:705-712

32. Klotz D, Schaefer C, Stavropoulou D, Fuchs H, Schumann S (2017) Leakage in nasal high-frequency oscillatory ventilation improves carbon dioxide clearance - a bench study. Pediatr Pulmonol 52(3):367-372

33. Hadj-Ahmed MA, Samson N, Nadeau C, Boudaa N, Praud JP (2015) Laryngeal muscle activity during nasal high-frequency oscillatory ventilation in non sedated newborn lambs. Neonatology. 107(3):199-205

Publisher's note Springer Nature remains neutral with regard to jurisdictional claims in published maps and institutional affiliations. 